\title{
Numerical Analysis of Two-Dimensional Viscoelastic Flow
}

\author{
By Kiyoji Nakamura and Akira Horikawa, Member, TMSJ \\ Department of Mechanical Engineering, Osaka University, Suita, Osaka
}

Based on the Journal of the Textile Machinery Society of Japan, Transaction, Vol. 29, No. 7, T92-99 (1976)

\begin{abstract}
The numerical analysis using the vorticity and the stream function is so useful to study two-dimensional viscous liquid flows that many problems have been solved by this method. In the present paper, this method has been expanded to two-dimensional viscoelastic liquids, and, as an example, the flow of a viscoelastic liquid into a slit has been calculated by using the Maxwell model.

Comparing the results of numerical calculations with those of experiments, we found that the former agreed well with the latter. From this fact, it is clear that this numerical analysis is valid for the study of viscoelastic liquid flows.

As it is easy to change the constitutive equations in this method, this method is applicable to the analysis of many viscoelastic flows.
\end{abstract}

\section{Introduction}

In analysing theoretically the flow of fluids, it is important and instructive to understand the characteristics of the mathematical equations governing the flows. Such equations are generally expressed in non-linear partial differential equations which cannot be solved analytically in many cases, and so the numerical analysis is the only way effective to solve flow problems successfully, many of which are Newtonian flows governed by the Navier-Stokes equation. However, there are many liquids not governed by the Navier-Stokes equation. These are called non-Newtonian liquids, which are more complicated than the Newtonian flow in expressing their characteristics mathematically. Hence, the numerical analysis is expected suitable in such studies.

Unfortunately, there has been very little literature available for the numerical analysis of non-Newtonian flows in spite of the urgent necessity. So we try in this paper a numerical study of a two-dimensional non-Newtonian flow, particularly of a two-dimensional viscoelastic flow into a slit.

\section{Fundamental Equations}

2.1 Equations involving vorticity and stress function

Fundamental equations for the flow of incompressible fluid are:

The equation of continuity;

$v_{i, i}=0$

The equation of motion;

$\rho\left(\frac{\partial v_{i}}{\partial t}+v_{j} v_{i}, j\right)=-p, i+T_{i \jmath}$,

where $v_{i}$ is a component of velocity vector, $T_{i j}$ a deviatoric component of stress tensor, $p$ an isotropic component of stress tensor, $\rho$ the density and $t$ the time.

If we assume the flow to be two-dimensional, we can introduce the stream function $\phi$ by

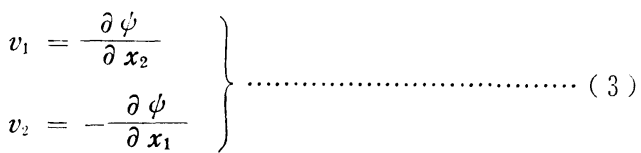

Using this stream function $\phi$, we can rewrite eqs. (1) and (2) in the form

$$
\begin{aligned}
& -\frac{\partial \Delta \psi}{\partial t}+\frac{\partial \psi^{\prime}}{\partial x_{1}} \frac{\partial \Delta \psi}{\partial x_{2}}-\frac{\partial \psi}{\partial x_{2}}-\frac{\partial \Delta \psi}{\partial x_{1}} \\
& =\frac{1}{\rho}\left\{\frac{\partial^{2}\left(T_{22}-T_{11}\right)}{\partial x_{1} \partial x_{2}}+\left(\frac{\partial^{2}}{\partial x_{1}^{2}}-\frac{\partial^{2}}{\partial x_{2}^{2}}\right) T_{12}\right\} \cdots \cdots(4)
\end{aligned}
$$

Now, when a relation between stress tensor $T_{i j}$ and velocity vector $v_{i}$ is given, a stream function $\phi$ is calculated from eq. (4) to obtain a flow field. It is difficult, however, to calculate eq. (4) numerically because this equation is non-linear in stream function $\phi$. Then, introducing vorticity $\omega$ as follows

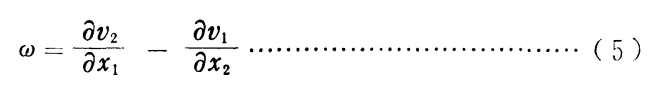

we obtain

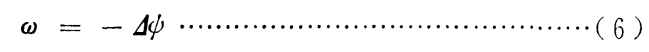

and can rewrite eq. (4) in the form

$$
\begin{aligned}
& \frac{\partial \omega}{\partial t}+\frac{\partial\left(v_{1} \omega\right)}{\partial x_{1}}+\frac{\partial\left(v_{2} \omega\right)}{\partial x_{2}} \\
& =\frac{1}{\rho}\left\{\frac{\partial^{2}\left(T_{22}-T_{11}\right)}{\partial x_{1} \partial x_{2}}+\left(\frac{\partial^{2}}{\partial x_{1}{ }^{2}}-\frac{\partial^{2}}{\partial x_{2}{ }^{2}}\right) T_{12}\right\} \cdots \cdots(7)
\end{aligned}
$$


These eqs. (6) and (7) are fundamental equations for numerical analysis.

2.2 Constitutive equation for viscoelastic fluids

While many different constitutive equations have been proposed for viscoelastic fluids, they may be divided into two broad groups; the rate type and the integral type. The present study will be restricted to the former type.

The rate type constitutive equation for fluids is given by the form ${ }^{[1]}$

$$
\begin{aligned}
& \widehat{T}_{i, j^{(s)}}=F_{i j}\left(T_{p q}, \widehat{T}_{p q}, \cdots, \widehat{T}_{p q}^{(s-1)} ; D_{k m},\right. \\
& \left.\cdots, D_{k m}{ }^{(r)} ; \rho\right)
\end{aligned}
$$

where Note 1)

$$
\begin{aligned}
& \widehat{T}_{i, i}=\frac{\partial T_{i, j}}{\partial t}+v_{k} T_{i j, k}-W_{i k} T_{k j}+T_{i k} W_{k, j} \cdots \cdots(g) \\
& D_{k m}=\frac{1}{2}\left(v_{k, m}+v_{m}, k\right) \cdots \cdots \cdots \cdots \cdots \cdots \cdots \cdots \cdots(10) \\
& W_{k \cdot m}=\frac{1}{2}\left(v_{k, m}-v_{m, k}\right) \cdots \cdots \cdots \cdots \cdots \cdots \cdots \cdots(11)
\end{aligned}
$$

Since eq. (8) is in a functional expression, it must be reduced to a specific function before a numerical calculation is carried out. Such specific models are proposed as Maxwell model, Oldroyd model ${ }^{[2,3]}$, Denn model ${ }^{[4]}$ etc. Substituting the relation between stress and velocity into eqs.(3), (6) and (7), we will obtain the flow field.

\section{Finite Difference Approximation of Fundamental Equations}

The equations relating to the flow field are eqs. (3), (6), (7) and (8). Let us solve these equations by the finite difference method.

When the flow field $v_{i}(t)$ at time $t \leqq t_{0}$ is given, we can calculate the flow field by the following procedure;

(i) calculating $T_{i j}\left(t_{0}\right)$ using eq. (8),

(ii) calculating $\omega\left(t_{0}\right)$ using eq. (5),

(iii) calculating $\omega\left(t_{0}+\Delta_{t}\right)$ using eq. (7),

(iv) calculating $\phi\left(t_{0}+\Delta_{t}\right)$ using Poisson's equation (6),

(v) calculating $v_{i}\left(t_{0}+\Delta_{t}\right)$ using eq. (3).

Once $v_{i}\left(t_{0}+\Delta_{t}\right)$ is obtained in the above manner, we can calculate $v_{i}\left(t_{0}+2 \Delta_{t}\right)$ by repeating $\left.\left.\mathrm{i}\right)-\mathrm{v}\right)$. The iterative process is terminated when $v_{i}$ converges, and this result is considered to be the stable flow field.

3.1 Location of the finite difference points of the variables relative to the mesh

In the fluid we specify a mesh of points at which the variables $v_{1}, v_{2}, \omega, T_{i j}$ and $\phi$ may be defined ${ }^{[5]}$. A representation of this is given in Fig. 1; the stream function $\phi$ is located on the mesh point and the velocity $v_{i}$ is located halfway between two adjacent mesh points. Namely,

$$
\begin{aligned}
& \left(v_{1}\right)_{I, J+1 / 2}=\frac{\phi_{I, J}-\psi^{\prime} I, J}{\Delta_{x 2}} \ldots \\
& \left(v_{2}\right)_{I+1 / 2, J}=\frac{\psi_{I, J}-\phi_{I+1}, J}{\Delta_{x 1}} .
\end{aligned}
$$

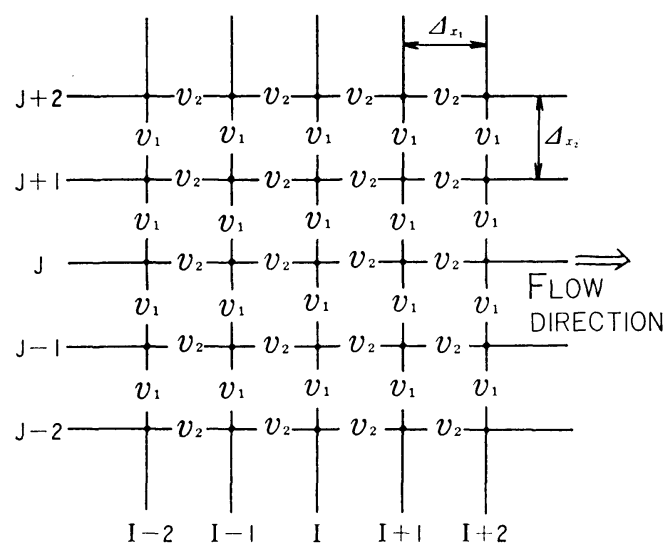

Fig. 1 Location of the finite difference points of the variables $\left(.=\psi, \omega, T_{i j}\right)$

$$
\begin{aligned}
& \left(v_{1}\right)_{I, J}=\frac{\left(v_{1}\right)_{I, J+1 / 2}+\left(v_{1}\right)_{I, J-1 / 2}}{2} \ldots \\
& \left(v_{2}\right)_{I, J}=\frac{\left(v_{2}\right)_{I+1 / 2, J}+\left(v_{2}\right)_{I-1 / 2, J}}{2}
\end{aligned}
$$

Vorticity $\omega$ and stress $T_{i j}$ are located on the mesh point as in the stream function $\psi$.

3.2 Finite difference approximation of Poisson's equation

The value of stream function $\phi_{I, J}$ at the $(I, J)$ mesh point is computed using the accelerated Liepmann method.

$$
\begin{aligned}
& \psi_{l, I^{(k+1)}}=\psi_{I, j^{(k)}}+\frac{\xi}{2\left(\Delta_{x 1^{2}}{ }^{2}+\Delta_{x 2^{2}}\right)}\left\{\Delta _ { x 2 } { } ^ { 2 } \left(\psi_{I+1}^{\prime} J^{(k)}\right.\right. \\
& +\phi_{I-1, l^{(k+1)}}+\Delta_{x 1^{2}}{ }^{2}\left(\phi_{I, I_{+1}}{ }^{(k)}+\phi_{I, I-1}{ }^{(k+1)}\right) \\
& \left.-2\left(\Delta_{x 1}{ }^{2}+\Delta_{x 2^{2}}{ }^{2}\right) \psi_{I, J^{\prime}}\left(k^{)}\right)+\Delta_{x 1}{ }^{2} \Delta_{x 2}{ }^{2} \omega_{l, J}\right\} \cdots
\end{aligned}
$$

where $k$ is the number of iteration step and $\xi$ is the overrelaxation factor. Criteria of the convergence of the stream function $\phi$ may be, for example,

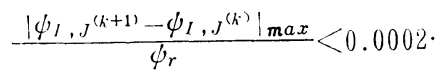

and

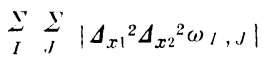

$$
\begin{aligned}
& \sum_{l} \sum_{J} 12\left(\Delta_{x 1^{2}}+\Delta_{x 2^{2}}{ }^{2}\right) \psi_{l, J}-\Delta_{x 2}{ }^{2}\left(\psi_{l+l, I}+\psi_{I-1, j}\right)
\end{aligned}
$$

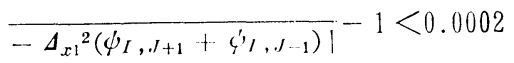

where $\phi_{r}$ is the value of the reference stream function.

3.3 Finite difference approximation of the vorticity equation (7)

Assume that the entire set of values of vorticity $\omega$, velocity $v_{i}$ and stress $T_{i j}$ at some time $t$. Then we may approximate $\omega$ at a small increment of time later, i.e. at $t+\Delta_{t}$, by a finite difference approximation of eq. (7): 


$$
\begin{aligned}
& \omega_{I, J}\left(t+\Delta_{t}\right)=\omega_{I, J}(t)-\Delta_{t}\left\{\frac{\left(v_{1} \omega\right)_{I+1}, J}{\Delta_{x 1}}(t)\right. \\
& \left.\frac{-\left(v_{1} \omega\right)_{I, J}(t)}{+}+\frac{\left(v_{2} \omega\right)_{I, J+1}(t)-\left(v_{2} \omega\right)_{I, J}(t)}{\Delta_{x 2}}\right\} \\
& +\frac{\Delta_{t}}{\rho}\left\{\frac{\left(T_{22}-T_{11}\right)_{I+1}, J+1}{\Delta_{x 1} \Delta_{x 2}}-\left(T_{22}-T_{11}\right)_{I, J+1}(t)\right. \\
& +\left(T_{22}-T_{11}\right)_{I, J}(t)-\left(T_{22}-T_{11}\right)_{I+1, J}(t) \\
& +\frac{\left(T_{12}\right)_{I+1}, J(t)-2\left(T_{12}\right)_{I, J}(t)+\left(T_{12}\right)_{I-1, J}(t)}{\Delta_{x 1}{ }^{2}} \\
& \left.-\frac{\left(T_{12}\right)_{I, J+1}(t)-2\left(T_{12}\right)_{I, J}(t)+\left(T_{12}\right)_{I, J-1}(t)}{\Delta_{x 2}{ }^{2}}\right\}
\end{aligned}
$$

3.4 Finite difference approximation of constitutive equation (8)

Assuming that the constitutive equation (8) is in linear relation to $T_{i j}, \hat{T}_{i j}, \ldots, \hat{T}_{i j}^{(\mathrm{s}-1)}$, the time derivative (9) is written in the form

$$
\begin{aligned}
& \left(\widehat{T}_{i j}\right)_{I, J}(t)=\frac{\left(T_{i j}\right)_{I, J}(t)-\left(T_{i j}\right)_{I, J}\left(t-\Delta_{t}\right)}{\Delta_{t}} \\
& +\left(v_{k} T_{i j, k}\right)_{I, J}\left(t-\Delta_{t}\right)-\left(W_{i k} T_{k j}\right)_{I, J}\left(t-\Delta_{t}\right) \\
& +\left(T_{i k} W_{k j}\right)_{I, J}\left(t-\Delta_{t}\right) \cdots \cdots \cdots \cdots \cdots \cdots \cdots \cdots \cdots \cdots \cdots \cdots \cdots \cdots \cdots \cdots \cdots \cdots \cdots \cdots \cdots \cdots
\end{aligned}
$$

Then, we can calculate $\left(\hat{T}_{i j}\right)_{I, J}(t)$ from the values at time $t$ and $t-\Delta_{t}$. Thus, eq. (8) may be written in the following form:

$$
\begin{aligned}
& \left(T_{i j}\right)_{I, J}(t)=G_{i j}\left\{\left(T_{p q}\right)_{I}, J\left(t-\Delta_{t}\right), \cdots,\right. \\
& \left(T_{p q}\right)_{I, J}\left(t-s \Delta_{t}\right) ;\left(D_{k_{m}}\right)_{I, J}(t), \cdots, \\
& \left.\left(D_{k m}\right)_{I, J}\left(t-r \Delta_{t}\right) ; \rho\right\}
\end{aligned}
$$

and stress values at time $t$ are obtained from both the stress and the deformation velocity at the past time.

\section{Application to a Viscoelastic Flow into a Slit}

Consider a two-dimensional viscoelastic converging flow shown in Fig. 2 as an example of the application of the

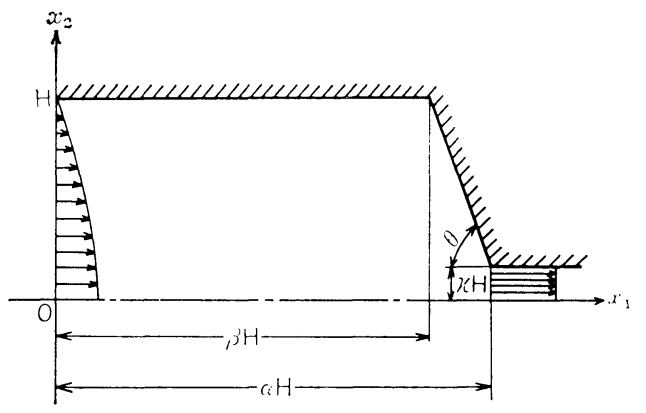

Fig. 2 Converging flow geometry above proposed numerical method.

We first of all assume as follows:

i) No body force,

ii) Incompressible and isothermal flow,

iii) A constant velocity in $x_{1}$ direction and zero velocity in $x_{2}$ direction at the exit.

iv) A fully-developed two-dimensional plane flow at the entrance $x_{1}=0$.

4.1 Fundamental flow equations

Introducing dimensionless quantities

$$
\begin{aligned}
t^{*} & =\frac{V}{H} t \\
x_{i} & =\frac{x_{i}}{H} \\
v_{i}^{*} & =\frac{v_{i}}{V} \\
T_{i j} & =\frac{H}{\eta V} T_{i j} \\
p^{*} & =\frac{H}{\eta V} p \\
\psi^{*} & =\frac{\psi}{H V} \\
\omega^{*} & =\frac{\omega}{H V}
\end{aligned}
$$

where $V$ and $H$ are the characteristic velocity and length respectively, we may rewrite eqs. (3), (5), (6) and (7) in the following forms;

$$
\begin{aligned}
& v_{1}^{*}=\frac{\partial \psi^{*}}{\partial x_{2} *} \\
& v_{2}^{*}=-\frac{\partial \psi^{*}}{\partial x_{1}^{*}} \\
& \omega^{*}=\frac{\partial v_{2}^{*}}{\partial x_{1}{ }^{*}}-\frac{\partial v_{1}^{*}}{\partial x_{2} *} \\
& \omega *=-\Delta \psi^{\prime} * \\
& \frac{\partial \omega^{*}}{\partial t^{*}}+\frac{\partial\left(v_{1}^{*} \omega^{*}\right)}{\partial x_{1}^{*}}+\frac{\partial\left(v_{2}^{*} \omega^{*}\right)}{\partial x_{2} *} \\
& =\frac{1}{R_{e}}\left\{\frac{\partial^{2}\left(T_{22}{ }^{*}-T_{11}^{*}\right)}{\partial x_{1} * \partial x_{2}{ }^{*}}+\left(\frac{\partial^{2}}{\partial x_{1}{ }^{* 2}}-\frac{\partial^{2}}{\partial x_{2}{ }^{* 2}}\right) T_{12} *\right\}
\end{aligned}
$$

where $\operatorname{Re}=\rho V H / \eta$.

Here, we further restrict the constitutive equation to the Maxwell model:

$$
T_{i j}+\lambda \widehat{T}_{i j}=2 \eta D_{i, i}
$$

This equation may be written by eq. (22) in the dimensionless form

$$
\begin{aligned}
& \left(T_{22} *-T_{11} *\right)+W_{e}\left\{\frac{\partial\left(T_{22} *-T_{11} *\right)}{\partial t^{*}}+\frac{\hat{\partial}\left\{v_{1}^{*}\left(T_{22} *-T_{11} *\right)\right\}}{\partial x_{1} *}+\frac{\partial\left\{v_{2} *\left(T_{22} *-T_{11} *\right)\right\}}{\partial x_{2} *}-2 \omega * T_{12} *\right\} \\
& =2\left(D_{22} *-D_{11} *\right) \\
& T_{12} *+W_{e}\left\{\frac{\partial T_{12} *}{\partial t *}+\frac{\partial\left(v_{1}^{*} T_{12}^{*}\right)}{\partial x_{1}^{*}}+\frac{\partial\left(v_{2}^{*} T_{12} *\right)}{\partial x_{2}^{*}}+\frac{1}{2} \omega *\left(T_{22} *-T_{11} *\right)\right\}=2 D_{12} *
\end{aligned}
$$


where $\lambda$ is relaxation time, $\eta$ is coefficient of viscosity and $W e=\lambda V / H$.

The finite difference equations of (28) and (29) become

$$
\begin{aligned}
& \left(1+\frac{W_{e}}{\Delta_{t}}\right)\left(T_{22} *-T_{11}\right)_{I, J}(t)=2\left(D_{22} *-D_{11} *\right)_{I, J}(t)+W_{e}\left\{\frac{\left(T_{22} *-T_{11} *\right)_{I, J}\left(t-\Delta_{t}\right)}{\Delta_{t}}-\frac{1}{\Delta_{x}}\left[\left\{v _ { 1 } * \left(T_{22} *\right.\right.\right.\right. \\
& \left.\left.-T_{11}^{*}\right)\right\}_{I+1, J}\left(t-\Delta_{t}\right)-\left\{v_{1}^{*}\left(T_{22} *-T_{11} *\right)\right\}_{I, J}\left(t-\Delta_{t}\right)+\left\{v_{2}^{*}\left(T_{22} *-T_{11} *\right)\right\}_{I, J+1}\left(t-\Delta_{t}\right)-\left\{v_{2}^{*}\left(T_{22} *-T_{11} *\right)\right\}_{I}, J \\
& \left.\left.\left(t-\Delta_{t}\right)\right]+2\left(\omega * T_{12}^{*}\right)_{I, v}\left(t-\Delta_{t}\right)\right\} \\
& \left(1+\frac{W_{e}}{\Delta_{t}}\right)\left(T_{12}\right)_{I, J}(t)=2\left(D_{12} *\right)_{I, J}(t)+W_{e}\left[\frac{\left(T_{12} *\right)_{I}, J\left(t-\Delta_{t}\right)}{\Delta_{t}}-\frac{1}{\Delta_{x}}\left\{\left(v_{1} * T_{12} *\right)_{I+1}, J\left(t-\Delta_{t}\right)-\left(v_{1} * T_{12} *\right)_{I, J}\right.\right. \\
& \left.\left.\left(t-\Delta_{t}\right)+\left(v_{2}^{*} T_{12} *\right)_{I, J+1}\left(t-\Delta_{t}\right)-\left(v_{2} * T_{12} *\right)_{I, J}\left(t-\Delta_{t}\right)\right\}-\frac{1}{2}\left\{\omega *\left(T_{22} *-T_{11} *\right)\right\}_{I, J}\left(t-\Delta_{t}\right)\right]
\end{aligned}
$$

where $\Delta_{x 1} *=\Delta_{x 2} *=\Delta_{x}$ is further assumed.

4.2 Boundary conditions

A. Boundary conditions of $v_{1}{ }^{*}$ and $v_{2} *$

$$
\begin{aligned}
& v_{1}^{*}=\frac{3}{2}\left(1-x_{2}{ }^{* 2}\right) \\
& v_{2}^{*}=0
\end{aligned}
$$

at $x_{1} *=0$ Note 2 )

ii) As the flow is symmetric with respect to $x_{1} *$ axis,

$$
\begin{aligned}
\frac{\partial v_{1}{ }^{*}}{\partial x_{2}{ }^{*}} & =0 \\
v_{2}{ }^{*} & =0
\end{aligned}
$$

at $x_{2} *=0$.

iii)

$$
\begin{aligned}
& v_{1} *=\frac{1}{\kappa} \\
& v_{2}^{*}=0
\end{aligned}
$$

at $x_{1} *=\alpha, 0 \leqq x_{2} * \leqq \kappa$

iv)

$$
v_{1}^{*}:=v_{2}^{*}=0
$$

at $0 \leqq x_{1} * \leqq \beta, x_{2}^{*}=1$

$$
v_{1}^{*}=v_{2}^{*}=0
$$

at

$$
\beta \leqq x_{1} * \leqq \alpha, x_{2} *=\frac{\kappa-1}{\alpha-\beta} x_{1}^{*}+1-\frac{\kappa-1}{\alpha-\beta} \beta
$$

B. Boundary conditions of $\phi^{*}$

Boundary conditions of $\psi^{*}$ are obtained from boundary conditions of $v_{1}^{*}$ and $v_{2} *$;

i)

$$
\phi^{*}=\frac{3}{2}\left(x_{2}^{*}-\frac{1}{3} x_{2}^{* 3}\right)
$$

at $x_{1}^{*}=0$

ii)

$$
\phi^{*}=0
$$

at $x_{2} *=0$

iii)

$$
\psi^{*}=-\frac{1}{\kappa} x_{2}^{*}
$$

at $x_{1} *=\alpha, 0 \leqq x_{2} * \leqq \kappa$

iv)

$$
\psi^{\prime *}=1
$$

at the wall.

C. Hypothetical points of $v_{1}{ }^{*}$ and $v_{2}{ }^{*}$

In order to calculate the values of $T_{22} *-T_{11} *, T_{12} *$ and $\omega^{*}$ at the wall, the values of $\partial v_{1} * / \partial x_{2}{ }^{*}$ and $\partial v_{2} * / \partial x_{1} *$ etc. are necessary. Since the values of $v_{1}^{*}$ and $v_{2} *$ are not specified at the wall, hypothetical points are introduced such that, in the case of the boundary shown in Fig. 3(a),

$$
\left(v_{1}^{*}\right)_{-\not 2}=\left(v_{1}^{*}\right)_{0}
$$

That is to say, $\left(v_{1}^{*}\right)_{-1 / 2}$ outside of the wall is made equal to zero to satisfy the boundary conditions (35) and (36). Similar rule applies to $v_{2}{ }^{*}$. In the case of Fig. 3(b), the value outside of the wall is assumed to be equal to $\left(v_{1} *\right)_{0}$. 4.3 Initial velocity field

To start the numerical calculation, an initial velocity field has to be assumed. As the initial velocity field is desirable to be as close as possible to the final velocity field, we assume such an initial velocity field as is given by Stokes' approximation of the Navier-Stokes equation under the boundary conditions of eqs. (43) and (44). (This solution does not satisfy $v_{2}^{*}=0$ at the wall.) This solution

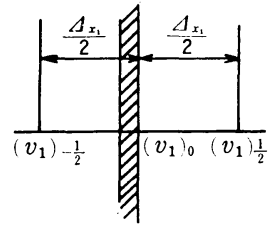

(a)

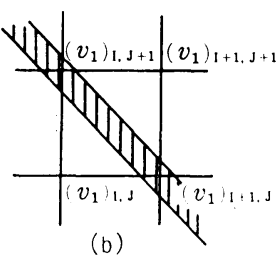

(b)
Fig. 3 Hypothetical point of $v_{1}$ 
is briefly described as follows: When the wall is oblique, the initial velocity field is identical to that in which velocities are zero in the region outside of the oblique wall.

In the Stokes' approximation, the stream function satisfies the biharmonic equation

$$
\nabla^{2} \nabla^{2} \psi^{*}=0
$$

Boundary conditions are assumed as shown in Fig. 4:

i)

$$
\begin{aligned}
& v_{1}^{*}=\frac{1}{\kappa}\left\{H\left(x_{2} *-1+\kappa\right)-H\left(x_{2} *-1-\kappa\right)\right\} \\
& v_{2}^{*}=0
\end{aligned}
$$

at

$$
x_{1} *=\frac{\alpha}{2}, \quad 0 \leqq x_{2} * \leqq 2
$$

where $H(x)$ is Heaviside's step function.

$$
\begin{aligned}
& \text { ii) } \\
& v_{1}^{*}=3 x_{2} *-\frac{3}{2} x_{2} * \quad \ldots \ldots \\
& v_{2}^{*}=0 \\
& x_{1}^{*}=-c_{2}^{c}, 0<x_{2}^{*} 2
\end{aligned}
$$$$
\text { at }
$$

Eqs. (43) and (44) can be expanded in Fourier series respectively as follows ${ }^{[8]}$ :

$$
\begin{aligned}
& v_{1}^{*}=\sum_{n=1}^{\sum} \frac{4}{n \pi \kappa} \sin \frac{n \pi}{2} \sin \frac{n \pi \pi}{2} \sin \frac{n \pi x_{2}{ }^{*}}{2} \ldots \\
& v_{1}^{*}=\sum_{n=1}^{\infty} \frac{24}{(n \pi)^{3}}\left\{1+(-1)^{n-1}\right\} \sin \frac{n \pi x_{2}{ }^{*}}{2}
\end{aligned}
$$

Now, assuming the solution in the form

$$
\phi^{*}=\sum_{n=1}^{\infty} \phi_{n}\left(\mu_{n,} x_{1} *\right) \cos \mu_{n} x_{2} *
$$

and substituting eq. (47) into eq. (42), we obtain

$$
\psi^{*}=\sum_{n=1}^{\infty}\left\{A_{n} \cosh \mu_{n} x_{1}^{*}+B_{n} x_{1} * \cosh \mu_{n} x_{1} *+C_{n} \sinh \mu_{n} x_{1} *+D_{n} x_{1} * \sinh \mu_{n} x_{1} *\right\} \cos \mu_{n} x_{2} * .
$$

where $\mu_{n}=n \pi / 2 . A_{n}, B_{n}, C_{n}$ and $D_{n}$ are derived from the boundary conditions as follows:

$$
\begin{aligned}
& A_{n}=-\frac{\left(\sinh \frac{\alpha \mu_{n}}{2}+\frac{\alpha \mu_{n}}{2} \cosh \frac{\alpha \mu_{n}}{2}\right)\left\{\frac{2}{\mu_{n} \kappa} \sinh \mu_{n} \sin \mu_{n} \kappa+\frac{3}{\mu_{n}{ }^{3}}\left(1+(-1)^{n-1}\right)\right\}}{\mu_{n}\left\{\alpha \mu_{n}+2 \sin \frac{\alpha \mu_{n}}{2} \cosh \frac{\alpha \mu_{n}}{2}\right\}} \\
& B_{n}=\frac{\cosh \frac{\mu_{n} \alpha}{2}\left\{\frac{2}{\mu_{n} \kappa} \sin \mu_{n} \sin \mu_{n} \kappa-\frac{3}{\mu_{n}^{3}}\left(1+(-1)^{n-1}\right)\right\}}{-\alpha \mu_{n}+2 \sinh \frac{\alpha \mu_{n}}{2} \cosh -\frac{\alpha \mu_{n}}{2}} \\
& C_{n}=\frac{\left(\cosh \frac{\alpha \mu_{n}}{2}+\frac{\alpha \mu_{n}}{2} \sinh \frac{\alpha \mu_{n}}{2}\right)\left\{\frac{2}{\mu_{n} \boldsymbol{\kappa}} \sin \mu_{n} \sin \mu_{n} \kappa-\frac{3}{\mu_{n}^{3}}\left(1+(-1)^{n-1}\right)\right\}}{\left\{\alpha \mu_{n}-2 \sinh \frac{\alpha \mu_{n}}{2} \cosh \frac{\alpha \mu_{n}}{2}\right\} \mu_{n}} \\
& D_{n}=\frac{\sinh \frac{\alpha \mu_{n}}{2}\left\{\frac{2}{\mu_{n} \kappa} \sin \mu_{n} \sin \mu_{n} \kappa+\frac{3}{\mu_{n}^{3}}\left(1+(-1)^{n-1}\right)\right\}}{\alpha \mu_{n}+2 \sinh \frac{\alpha \mu_{n}}{2} \cosh \frac{\alpha \mu_{n}}{2}}
\end{aligned}
$$

4.4 Results of numerical calculation and its discussion

Figs. 5 and 6 show calculated results for two different Weissenberg numbers, $5 \times 10^{-5}$ and $5 \times 10^{-4}$, under the same Reynolds number $2 \times 10^{-6}$. In this calculation, $\Delta_{x}$

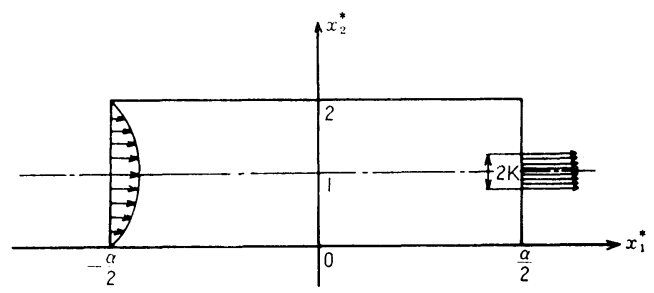

Fig. 4 Converging flow geometry for initial velocity was equal to $1 / 20, \Delta_{t}$ was equal to $R e W e$ and the number of time steps for convergence was about 4500. Eq. (17) was used as the criterion of convergence. Since Reynolds number was held constant in this calculation, the characteristic velocity $V$ may be considered not to change. Hence, the change of Weissenberg number is equivalent to change the relaxation time of the viscoelastic fluid. Figs. 5 and 6 show that the circulation flow enlarges as Weissenberg number increases, i.e. as the relaxation time increases. Fig. 7 shows the velocity field $v_{1}^{*}$ at $R e=2 \times 10^{-6}$ and $W e=5 \times 10^{-4}$. Moreover, Figs. 5 and 6 show that the velocity of circulating flow is much smaller than that of main flow. In comparison of the above numerical results with the experimental results ${ }^{[7]}$, tendencies agree well. 


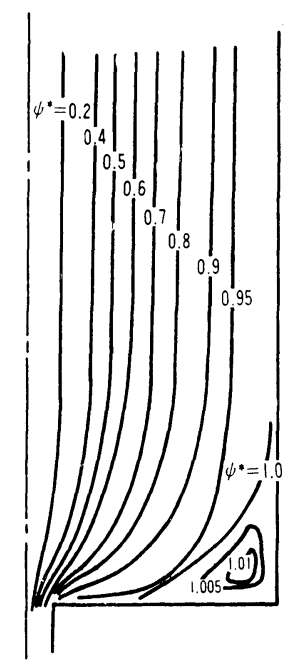

Fig. 5 Stream function $\phi^{*}$ (Reynolds number $2 \times 10^{-6}$, Weissenberg number $5 \times 10^{-5}$ )

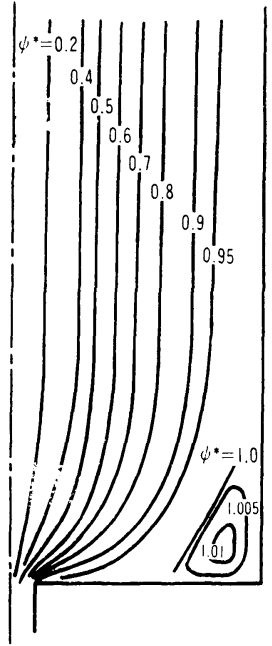

Fig. 6 Stream function $\phi^{*}$ (Reynolds number $2 \times 10^{-6}$, Weissenberg number $5 \times 10^{-4}$ )
Fig. 8 shows experimental results of flow patterns, which coicide with stream lines except near a U-shaped central region; calculated results are similar to the experimental results. Fig. 8(b) shows a flow pattern having large circulating flows. In this case the shape of the main flow lines looks like a wine-glass, but we failed to obtain this shape by the numerical calculation.

Fig. 9 shows the calculated stream line for the case of the oblique exit wall angle $60^{\circ}$ under the same Reynolds number and Weissenberg number as Fig. 6. In comparison

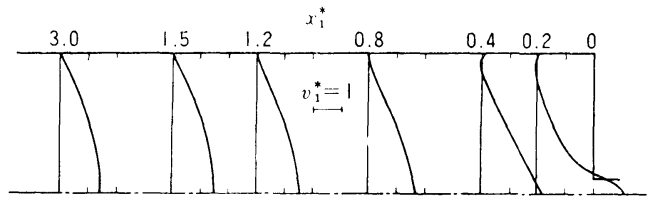

Fig. 7 Velocity distribution

(Reynolds number $2 \times 10^{-6}$, Weissenberg number $5 \times 10^{-4}$ )

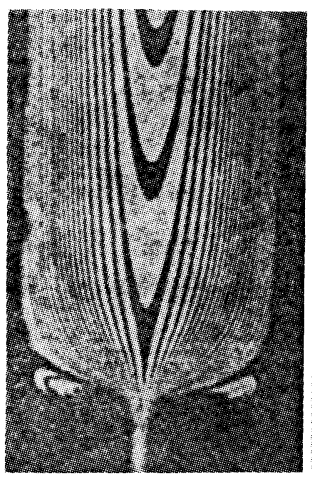

(a)

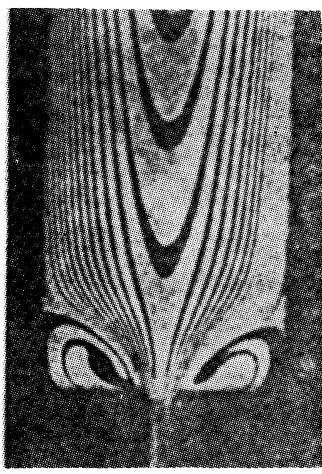

(b)
Fig. 8 Flow pattern obtained by experiment

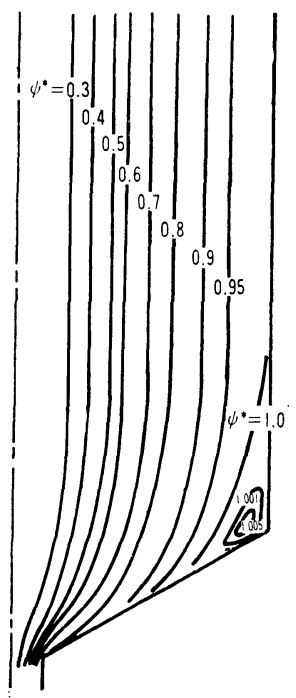

Fig. 9 Stream function $\psi^{*}$ (Reynolds number $2 \times 10^{-6}$, Weissenberg number $5 \times 10^{-4}$ and oblique wall angle $60^{\circ}$ )

of Figs. 6 and 9, it is seen that the circulating region becomes small in proportion to this oblique angle, and this result agrees with experimental results.

\section{Conclusions}

We proposed a numerical method for the two-dimensional viscoelastic flow, and calculated a flow into a slit using Maxwell model. In comparison of numerical results with experimental results the tendency agrees well. From this fact, this numerical method will be effective for the study of viscoelastic flow. Moreover, we can easily change the constitutive equation in this method.

Note 1) $\hat{T}_{i j}$ is called Jaumann derivative. Other derivatives may be used if they satisfy the material objectivity. Material derivative is used for $\mathbf{D}_{k m}$.

Note 2) If the flow channel is long in $x_{1}$ * direction, the influence of velocity distribution at $x_{1} *=0$ in the inlet region of the slit becomes small.

\section{References}

[1] C. Truesdell and W. Noll; The Non-Linear Field Theories of Mechanics, Springer-Verlag, Berlin (1965).

[2] J. G. Oldroyd, Proc. Roy. Soc. Lond., A200, 523(1950). [3] J. G. Oldroyd, Proc, Roy. Soc. Lond., A245, 278(1958).

[4] M. M. Denn, Chem. Eng. Sci., 22, 395(1967).

[5] J. Fromm, Method in Computational Physics, Academic Press, New York (1964).

[6] T. Akasaka, Suchikeisan (in Japanese), Korona-sha (1967).

[7] A. Horikawa, K. Nakamura and S. Umegaki, Sen'ikikai-gakkai Ronbunshu (in Japanese), 29, No. 5, 41 (1976).

[8] S. Moriguchi, K. Udagawa and S. Hitotsumatsu, Sugakukoshiki II (in Japanese), Iwanami-shoten (1957). 